\title{
Correction to: Surveying the arable plant diversity of conventionally managed farmland: a comparison of methods
}

\author{
Alexander Wietzke (D) Christoph Leuschner
}

Published online: 9 March 2021

(C) Springer Nature Switzerland AG 2021

\section{Correction to: Environ Monit Assess (2020) 192: 98 https://doi.org/10.1007/s10661-019- 8042-7}

The authors of the above-mentioned publication noticed two minor typing errors in the Electronic supplementary material file "Online Resource 10 Raw data" which have been corrected. The total herbaceous species number of one observed Edge_500 plot and the estimated total herbaceous species number of one observed field (plot type "Total") have each changed by 1 (Edge_500: 34 species instead of 35 species; "Total": 30 instead of 29 species). Online Resource 10 and the related Online Resources 7 and 8 have been updated in the Electronic supplementary materials of the published article. Owing to a calculation error, the analysis regarding the high-naturevalue species has also been updated. The following minor corrections resulted from the adaptations mentioned above:

The original article can be found online at https://doi. org/10.1007/s10661-019-8042-7.

A. Wietzke $(\bowtie) \cdot$ C. Leuschner

Department of Plant Ecology and Ecosystems

Research, University of Goettingen, Untere Karspüle 2,

37073 Goettingen, Germany

e-mail: alexander.wietzke@biologie.uni-goettingen.de

C. Leuschner

e-mail: cleusch@gwdg.de
- The following median values mentioned in the text of the published article changed minimally: The proportion of the estimated total herbaceous species number per field detected by Edge_500 plots changed to median $70.8 \%$ (instead of median $71.1 \%$; all crops pooled; Online Resource 2). The proportion of the estimated total herbaceous species number per field detected by Corner plots changed to median $44.6 \%$ (instead of median 44.7\%; all crops pooled; Online Resource 2). For wheat fields, the proportion of the estimated total herbaceous species number per field detected by Edge_500 plots changed to median $69.4 \%$ (instead of median $70.5 \%$ ). The proportion of the estimated typical arable plant species number per field detected by Corner plots changed to median $45.2 \%$ (instead of median $45.8 \%$; all crops pooled; Fig. 2d). The proportion of the estimated highnature-value species number per field detected in the plots Edge_500, Corner, Subplots, Edge_50 and Interior changed to median 77.8, 44.4, 28.6, 27.3 and $7.1 \%$, respectively (instead of median 75.0, 46.2, 30.8, 25.0 and 6.7\%; all crops pooled; Online Resource 3).

- Owing to the corrections in Online Resource 10 mentioned above, Fig. 2a, c and d), Online Resource 2 and Online Resource 3 needed to be updated (see below). The corrections did not lead to any changes regarding the significant differences between the plot types. 

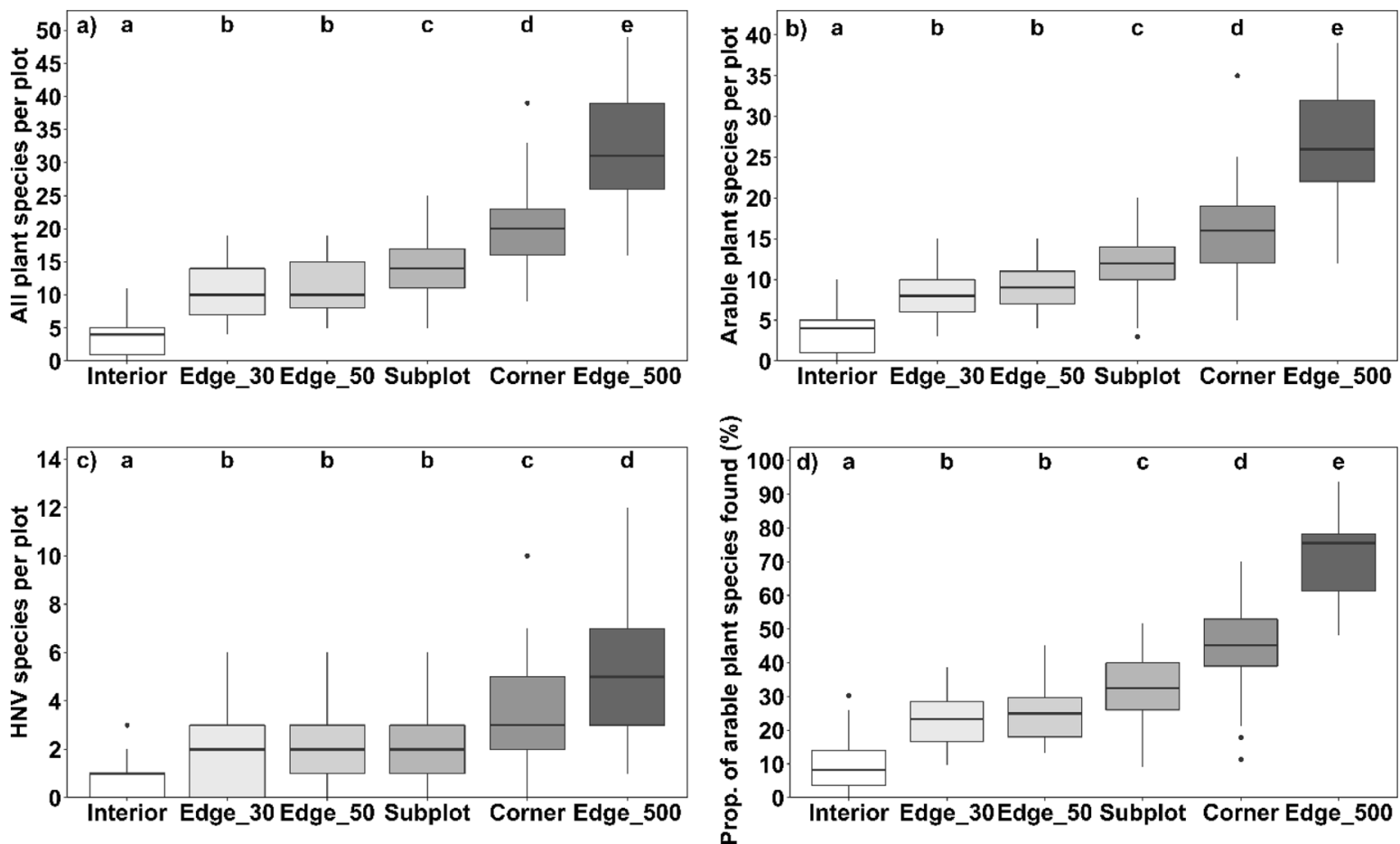

Fig. 2 Total number of herbaceous plant species (all plant species, woody seedlings and crops excluded) (a), number of arable plant species sensu stricto (as listed by Hofmeister and Garve 2006) (b), and number of high-nature-value species (HNV according to the German Federal Agency for Nature Conservation 2018) (c) in plots surveyed with six different methods. (d) Proportion (in \%) of the number of arable plant

- Fig. 3 and Online Resource 4, which show the relationship between the recorded plant species number in plots of the six survey methods and the estimated total plant species number in the respective field (referring to all herbaceous plants and arable plants sensu stricto), have been also updated owing to the corrections mentioned above (see below). These updates did not lead to any changes in the calculated correlation coefficients. With regard to the stated p-values of the correlations, there was only a minimal change in the $\mathrm{p}$-value concerning the correlation for the survey method Interior in Fig. 3 ( $p=0.86$ instead of $p=0.85)$. All other $\mathrm{p}$-values of the correlations stated in Fig. 3 and Online Resource 4 did not change.

In addition, a calculation error has been corrected regarding the mixed effects models dealing

species sensu stricto present in the field that is found in plots of the six survey methods. All data are averages over wheat, maize and rapeseed fields. Black lines in boxplots represent medians; $n=45$ ( 3 crop types $\times 15$ replicates per crop for each survey method), Tukey's test, $\alpha \leq 0.05$ : different small letters indicate significant differences between survey methods

with the influence of the plot type on the proportion of species found in a field. For the correct calculation, the function used for the response variable, which calculates the ratio of the number of species found in a plot to the estimated total number of species in a field, must be cbind("number of species found in the plot", "total number of species in the respective field" minus "number of species found in the plot") instead of cbind("number of species found in a plot", "total number of species in the respective field"). To account for overdispersion in two mixed effects models, an observation level random effect was included. The correction of the mixed effects models did not lead to any changes in the significant differences found between the plot types. As the mixed effects models were only used for the significance test, this correction also had no effect on the related figures of the published article (Fig. 2d, Online Resource 2 and Online Resource 3 are based 
Fig. 3 Number of arable plant species found in plots of the six survey methods in relation to total arable plant species number in the respective field; $R=$ Spearman's rank correlation coefficient with p-values; $n=45$ (per survey method)

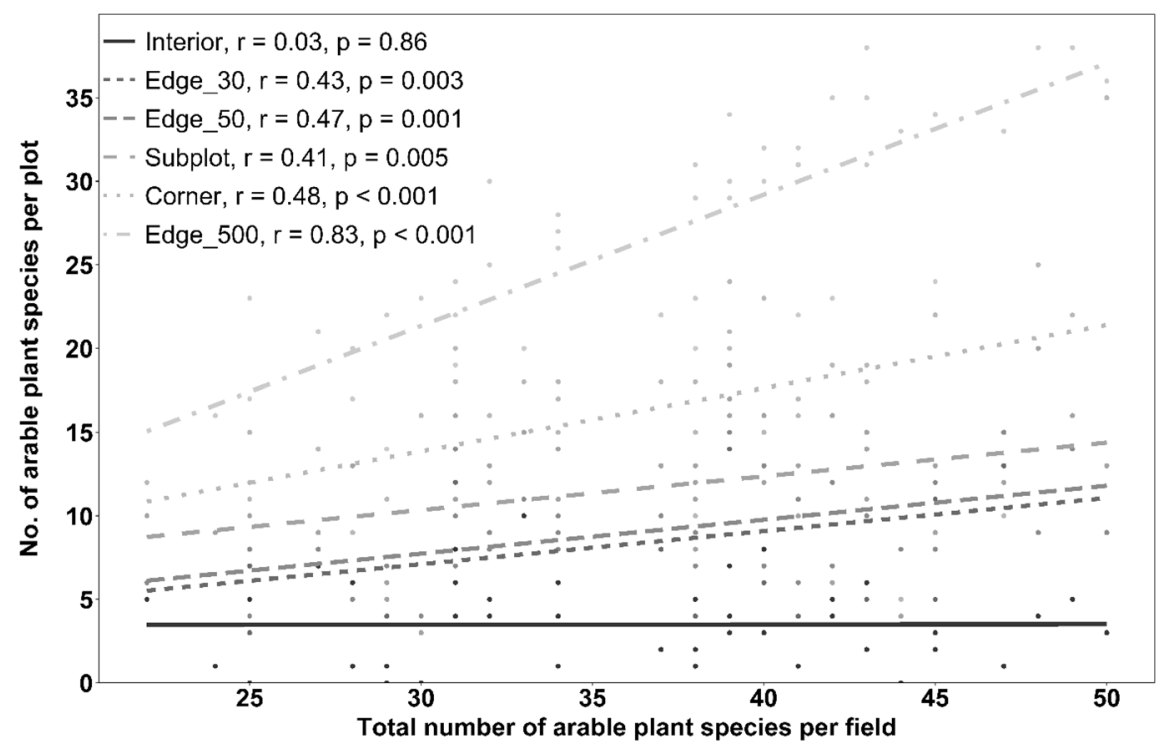

The authors apologize for the corrections that have become necessary and declare that these adaptions do not change the scientific conclusions drawn in any way. on the correctly calculated percentage ratios from the original data). The updated Online Resource 9 "Overview of used models and statistical results" is attached as an appendix file to this corrigendum.

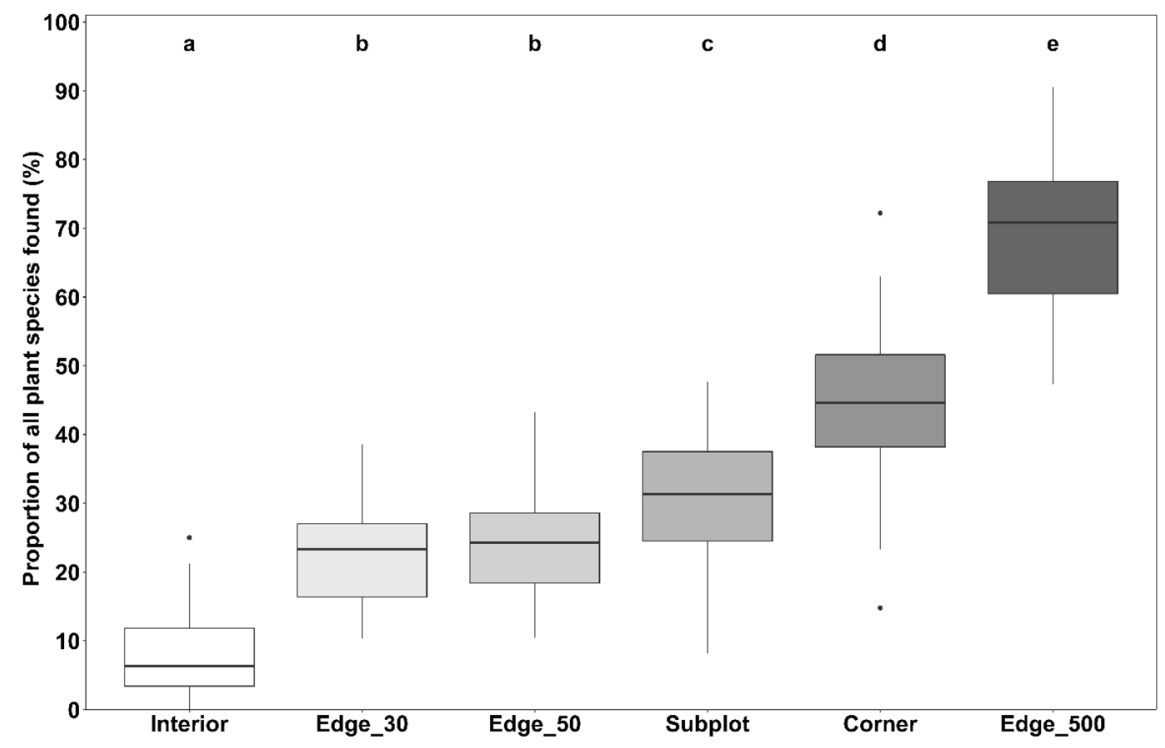

Online Resource 2 Proportion of the species number found in the plots $(\%)$ of the different survey methods relative to the field's total species pool (total perimeter count; all herbaceous species considered, crops and woody seedlings excluded; winter wheat, winter rapeseed and maize were pooled); Interior
$(50 \times 2 \mathrm{~m})$, Edge_30 $(30 \times 2 \mathrm{~m})$, Edge_50 $(50 \times 2 \mathrm{~m})$, Subplots (four plots of $5 \times 1 \mathrm{~m})$, Corner $(50 \times 2 \mathrm{~m})$ and Edge_500 $(500 \times 1 \mathrm{~m})$; black lines in boxplots represent medians; $n=45$ (per survey method), Tukey's test $\alpha \leq 0.05$ : different small letters indicate significant differences between survey methods 


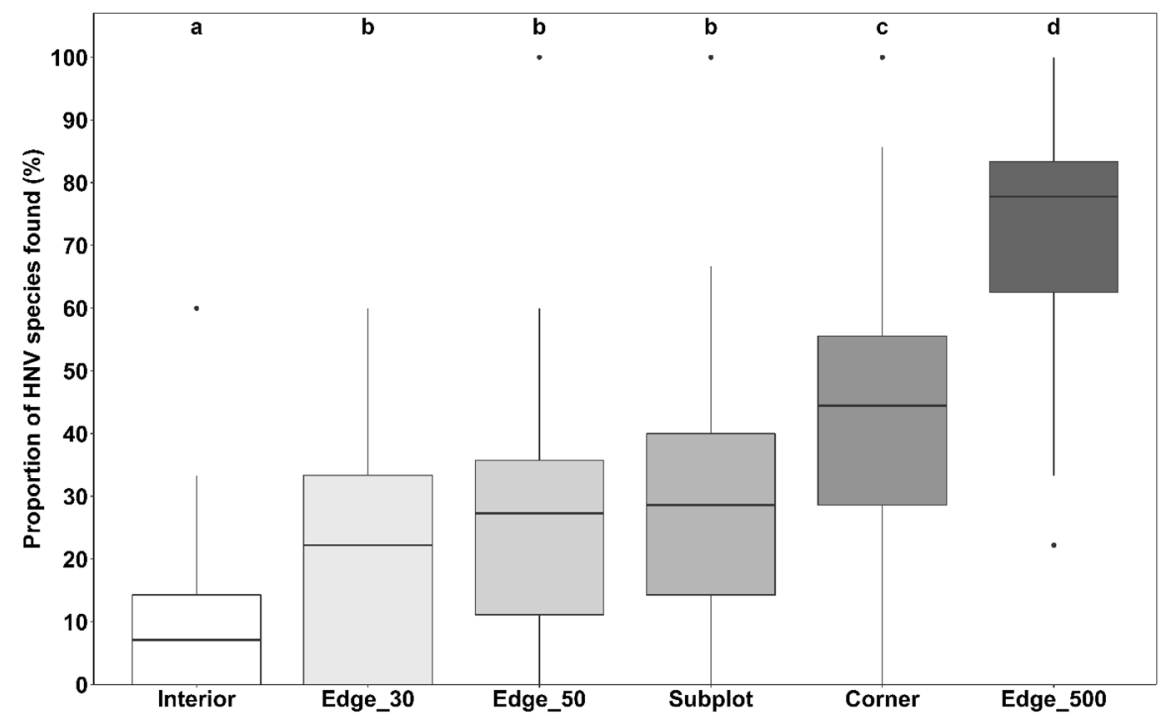

Online Resource 3 Proportion of the number of high-naturevalue (HNV; according to German Federal Agency for Nature Conservation, 2018) species (\%) found in the plots of the different survey methods relative to the field's total high-naturevalue (HNV) species pool (total perimeter count; winter wheat, winter rapeseed and maize were pooled); Interior $(50 \times 2 \mathrm{~m})$,
Edge_30 $(30 \times 2 \mathrm{~m})$, Edge_50 $(50 \times 2 \mathrm{~m})$, Subplots (four plots of $5 \times 1 \mathrm{~m})$, Corner $(50 \times 2 \mathrm{~m})$ and Edge_500 $(500 \times 1 \mathrm{~m})$; black lines in boxplots represent medians; $n=45$ (per survey method), Tukey's test $\alpha \leq 0.05$ : different small letters indicate significant differences between survey methods
Online Resource 4 Number of all herbaceous plant species found in plots of the six survey methods in relation to the field's total herbaceous species number (crops and woody seedlings excluded); survey method (see legend): Interior $(50 \times 2 \mathrm{~m})$, Edge_30 $(30 \times 2 \mathrm{~m})$, Edge_50 $(50 \times 2 \mathrm{~m})$, Subplots (four plots of $5 \times 1 \mathrm{~m}$ ), Corner $(50 \times 2 \mathrm{~m})$ and Edge_500 $(500 \times 1 \mathrm{~m}) ; R=$ Spearman's rank correlation coefficient with p-values; $n=45$ (per survey method)

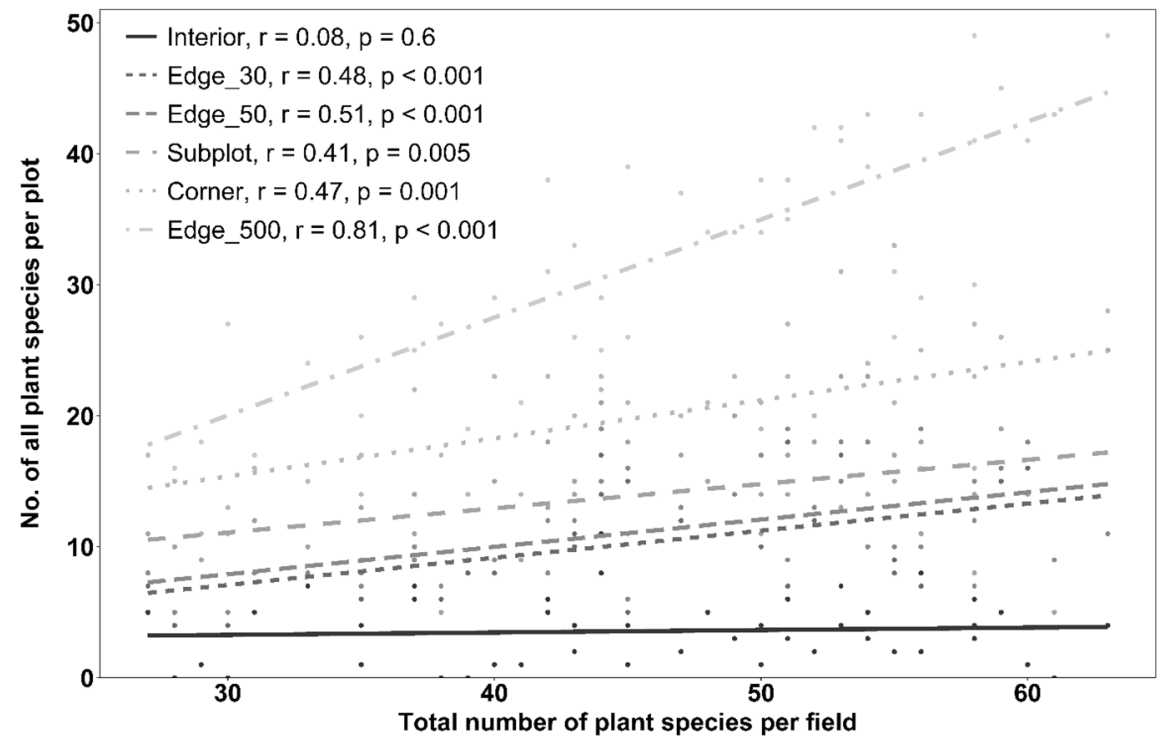

Publisher's Note Springer Nature remains neutral with regard to jurisdictional claims in published maps and institutional affiliations. 